\title{
Morphine Sulfate
}

National Cancer Institute

\section{Source}

National Cancer Institute. Morphine Sulfate. NCI Thesaurus. Code C669.

The sulfate salt of morphine, an opiate alkaloid isolated from the plant Papaver somniferum and produced synthetically. Morphine binds to and activates specific opiate receptors (delta, mu and kappa), each of which are involved in controlling different brain functions. In the central nervous and gastrointestinal systems, this agent has widespread effects including analgesia, anxiolysis, euphoria, sedation, respiratory depression, and gastrointestinal system smooth muscle contraction. 